[0212-7199 (2003) 20: 4; pp 179-182] ANALES DE MEDICINA INTERNA Copyright @ 2003 ARAN EDICIONES, S.L.

AN. MED. INTERNA (Madrid) Vol. 20, N. ${ }^{\circ} 4$, pp. 179-182, 2003

\title{
Utilidad de los medios de crecimiento para anaerobios en las bacteriemias de origen extrahospitalario
}

\author{
J. M. RUIZ-GIARDÍN, M. C. DEL REY ROMÁN'', M. SERRANO LÓPEZ', \\ G. GARCÍA MELCÓM, P. GONZÁLEZ RUANO, T. ISASIA MUÑOZ
}

Servicios de Medicina Interna-Urgencias y de ${ }^{1}$ Microbiología. Hospital Universitario de La Princesa. Madrid

\begin{abstract}
UTILITY OF ANAEROBIC BLOOD CULTURES IN EXTRAHOSPITALARY BACTEREMIAS
\end{abstract}

\section{RESUMEN}

Objetivo: Comparar rentabilidad de extracción de hemocultivos en medios para aerobios frente a anaerobios en bacteriemias diagnosticadas en urgencias, con intención de valorar la utilidad de los medios de cultivo para anaerobios.

Metodología: Durante un mes se realizaron hemocultivos a todos los pacientes que por criterio médico lo precisaban. Tres extracciones por sujeto en sitios de venopunción diferentes. En cada extracción se obtenían $10 \mathrm{ml}$ (distribuidos en $5 \mathrm{ml}$ para frasco aerobios, $5 \mathrm{ml}$ para frasco de aerobios). Posteriormente se compararon los frascos de aerobios frente a anaerobios. Se valoraron por tres clínicos todas las historias de los pacientes con hemocultivo positivo, clasificándolos como negativos, contaminantes o significativos.

Resultados: Se obtuvieron hemocultivos de un total de 180 pacientes. Se analizaron 152 grupos de hemocultivos (3 extracciones aerobiosanaerobios), sin valorarse los 28 restantes por no seguir el protocolo de estudio $24(13,3 \%)$; o por no haber podido analizar la historia clínica 4 $(2,2 \%)$. Grupo de tres extracciones sólo aerobios: Negativos 91 $(59,86 \%)$, contaminantes $36(23,68 \%)$, verdadero positivo en por lo menos una de las tres con o sin contaminante 25 (16,44\%). Grupo de tres extracciones sólo anaerobios: Negativos 127 (83,5\%), contaminantes 2 $(1,31 \%)$; verdadero positivo en por lo menos una de las tres con o sin contaminante $23(15,13 \%)$. Existen 6 casos $(8.6 \%)$ de verdaderos positivos en frasco de anaerobios que han sido negativos en los frascos de aerobios y que corresponden a los siguientes gérmenes: $3 \mathrm{E}$. coli; $1 \mathrm{~K}$. pneumoniae; 1 Peptostreptococcus sp; 1 Bacteroides sp y sobre el total de verdaderos positivos (31) suponen el 19,35\%. Las dos bacteriemias por anaerobios, fueron sospechadas clínicamente. El germen que con más frecuencia fue contaminante en medio de crecimiento para aerobios, es el Staphylococcus sp coagulasa negativo, 25 casos $(69,44 \%$ de todos los contaminantes).El germen productor de bacteriemia verdadera más frecuente fue el $E$. coli (también en anaerobios) con 12 casos $(38,70 \%$ de todas las verdaderas bacteriemias), seguido del Streptococcus pneumoniae, 4 casos $(12,90 \%)$

Conclusiones: Nuestra recomendación es mantener los cultivos en frascos de anaerobios en las sospechas de bacteriemia puesto que la rentabilidad global de los hemocultivos aumenta de forma significativa.

PALABRAS CLAVE: Hemocultivos. Bacteriemia. Anaerobios.

\section{ABSTRACT}

Objetive: To compare the rentability of anaerobic hemocultures with aerobic cultures in patients suffering bacteremia, trying to analize the rentability of anaerobic cultures.

Methods: There were analized all the hemocultures taken by medical decision for one month. There were taken three extractions by patient in different venopunctures points. In each extraction was taken $10 \mathrm{ml}(5 \mathrm{ml}$ in aerobic bottle, and $5 \mathrm{ml}$ in anaerobic bottle). There were compared microorganism isolated in aerobic bottles and anaerobic bottles. Three physician analized all the clinical dates of the patients with positive hemocultures and classified them as negatives, contaminants and significant.

Results: There were taken hemocultures of 180 patients. There were analyzed 152 hemocultures groups (3 extractions aerobic-anaerobic), without studying 28 owe to not following the study protocol $24(13.3 \%)$ or because of not possibility of studying clinical story 4 (2.2\%). Aerobic extractions: Negatives 91 (59.86\%), contaminants $36(23.68 \%)$, significant with or without contaminant 25 (16.44\%). Anaerobic extractions: Negatives $127(83.5 \%)$, contaminants $2(1.31 \%)$; significant with or without contaminant $23(15.13 \%)$. There are $6(8.6 \%)$ significant positive isolated in anaerobic bottles, that were negative in aerobic bottles, and are: $3 \mathrm{E}$. coli, $1 \mathrm{~K}$. pneumoniae, 1 Peptostreptococcus sp, 1 Bacteroides $\mathrm{sp}$, and over the total significant isolated (31) are the $19.35 \%$. Both anaerobic bacteremias were clinically suspected. Contaminant microorganism more frequently isolated in aerobic bottles was Staphylococcus $s p$, 25 cases (69.44\% of all contaminants). Significant microorganism more frequently isolated was E. coli (in anaerobic bottles too) in 12 cases $(38.70 \%)$ of all significant isolated, following by Streptococcus pneumoniae with 4 cases $(12.9 \%)$

Conclusions: We recommend collection of one aerobic and one anaerobic blood culture bottle per blood culture set because the global rentability of hemocultures is increased significantly.

KEY WORDS: Hemocultures. Bacteremia. Anaerobic.

Ruiz-Giardín JM, Del Rey Román MC, Serrano López M, García Melcóm G, González Ruano P, Isasia Muñoz T. Utilidad de los medios de crecimiento para anaerobios en las bacteriemias de origen extrahospitalario. An Med Interna (Madrid) 2003; 20: 179-182.

Trabajo aceptado: 9 de diciembre de 2002

Correspondencia: José Manuel Ruiz Giardín. Servicio de Medicina Interna-Urgencias. Hospital Universitario de La Princesa. C/ Diego de León, 62. 28006 Madrid. e-mail: chruiz@terra.es 


\section{INTRODUCCIÓN}

En el momento actual no existe uniformidad en cuanto a la determinación de la necesidad de realizar hemocultivos en medios de crecimiento para anaerobios, ya que están disminuyendo las bacteriemias por anaerobios, y están incrementándose las fungemias. Esto ha llevado a algunos autores a plantearse la supresión de los medios de cultivos para anaerobios (1), o a realizarlos únicamente en caso de sospecha clínica de infección por los mismos (2,3). Frente a esta tendencia, existen otros autores que abogan por mantener dichos cultivos, basándose en el hecho de que las bacteriemias por anaerobios no son predecibles (4), y que además existe crecimiento de gérmenes aerobios-anaerobios facultativos (5), incrementándose la rentabilidad de los hemocultivos.

El objetivo de nuestro estudio es analizar la rentabilidad de los medios de cultivo para anaerobios frente a los medios de cultivo para aerobios, en todas las bacteriemias clínicamente significativas recogidas en el servicio de urgencias en pacientes a los que por criterio médico se extrajeron hemocultivos, con intención de determinar la utilidad de los mismos.

\section{MATERIAL Y MÉTODOS}

Durante un mes se analizaron todos los hemocultivos que por criterio médico (síndrome febril, sospecha de sepsis con hipotermia, sospecha de infección endovascular con o sin fiebre)se extrajeron en el Servicio de Urgencias del hospital. A cada paciente se le realizaron tres extracciones en sitios de venopunción diferentes. En cada una de las tres extracciones se obtenían $10 \mathrm{ml}$ (distribuidos en $5 \mathrm{ml}$ para frasco de aerobios, $5 \mathrm{ml}$ para frasco de anaerobios). El sistema de hemocultivos empleado durante el periodo de estudio fue el Bactec 9240 (Becton and Dickinson, EE.UU.).Posteriormente se compararon los crecimientos de los frascos de aerobios frente a los de anaerobios. Se valoraron por tres clínicos todas las historias de los pacientes con hemocultivo positivo, clasificándolos como negativos, contaminantes o positivos verdaderos. Para el estudio comparativo aerobio-anaerobio, se analizaron sólo los hemocultivos verdaderos positivos, es decir los hemocultivos con valor clínico, independientemente de que se asociaran con crecimiento concomitante de gérmenes catalogados como contaminantes. Con los resultados se calculó un índice kappa, así como estudio de proporciones con los intervalos de confianza para el $95 \%$, partiendo del hecho de que la distribución muestral cumple los criterios para seguir una ley normal .La significación estadística de la diferencia entre las proporciones aerobios-anaerobios, se ha calculado con el test de Chi cuadrado. Igualmente se analizó el foco de origen en los casos de crecimiento de gérmenes anaerobios.

\section{RESULTADOS}

Se obtuvieron hemocultivos de un total de 180 pacientes. Se analizaron 152 grupos de hemocultivos (3 extracciones aerobos-anaerobios), no habiéndose valorado los 28 restantes por no haber seguido el protocolo de estudio 24 (13,3\%); o por no haber podido analizar la historia clínica 4 (2,2\%).

Analizando globalmente todos los grupos de hemocultivos, existen 31 bacteriemias verdaderas, de las cuales $17(54,83 \%)$ han crecido simultáneamente en frasco de aerobios y anaerobios, $8(25,80 \%)$ sólo en frasco de aerobios, y 6 (19,35\%) sólo en frasco de anaerobios. Grupo de tres extracciones sólo aerobios: Negativos $91 \quad(59,86 \%)$, contaminantes 36 $(23,68 \%)$, verdadero positivo en por lo menos una de las tres con o sin contaminante $25(16,44 \%)$. El germen que con más frecuencia fue contaminante en medio de crecimiento para aerobios, es el Staphylococcus sp coagulasa negativo, 25 casos $(69,44 \%$ de todos los contaminantes).El germen productor de bacteriemia verdadera más frecuente fue el E. coli (también en anaerobios) con 12 casos $(38,70 \%$ de todas las verdaderas bacteriemias), seguido del neumococo, 4 casos $(12,90 \%)$. Del conjunto de todas las bacteriemias, 2 (6,45\%), fueron bacteriemias polimicrobianas. Existen 8 bacteriemias verdaderas con crecimiento sólo en frasco de aerobios y no de anaerobios, que sobre el total de bacteriemias verdaderas 31 , suponen el $(25,8 \%)$.

Grupo de tres extracciones sólo anaerobios: Negativos $127(83,5 \%)$, contaminantes $2(1,31 \%)$; verdadero positivo en por lo menos una de las tres con o sin contaminante 23 $(15,13 \%)$. Existen 6 casos $(8,6 \%)$ de verdaderos positivos en frasco de anaerobios que han sido negativos en los frascos de aerobios y que corresponden a los siguientes gérmenes: $3 \mathrm{E}$. coli; $1 \mathrm{~K}$. pneumoniae; 1 Peptostreptococcus sp; 1 Bacteroides $s p$ y sobre el total de verdaderos positivos (31) suponen el $19,35 \%$. Las dos bacteriemias por anaerobios, fueron sospechadas clínicamente, un paciente con absceso en cavidad oral, y un paciente con neoplasia intraabdominal y fiebre. La clasificación por gérmenes se encuentra en la Tabla I. La medida de acuerdo entre el medio de crecimiento de aerobios y anaerobios, índice kappa es de $0,557 \mathrm{p}<0,001$. La proporción de hemocultivos verdaderos positivos dentro del global de los verdaderos positivos en frasco de aerobios, es del $80 \%$ con un intervalo de confianza al $95 \%$ entre $65-94 \%$, siendo en frasco de anaerobios del $74 \%$ con un intervalo de confianza al $95 \%$ entre $58-89 \%$. La diferencia entre ambas proporciones aerobios-anaerobios presenta significación estadística calculada con el test de Chi cuadrado con una $\mathrm{p}<0,001$. La diferencia entre las proporciones aerobios, y aerobio-anaerobio (de los hemocultivos verdaderos positivos), presenta igualmente significación estadística con una $\mathrm{p}<0,001$.

\section{DISCUSIÓN}

En este estudio llaman la atención dos datos: en primer lugar la presencia de 6 episodios de bacteriemias verdaderas (19,35\% del total 31 episodios) con crecimiento sólo en frasco de anaerobios y que no habrían podido ser identificados si no se hubieran realizado hemocultivos en medio para anaerobios. Este dato independientemente de su significación estadística, tiene un importante valor clínico puesto que su no realización habría supuesto la mencionada pérdida de 6 bacteriemias verdaderas. Frente a este dato cabría plantearse la pregunta de si el diagnóstico de dichas bacteriemias supuso modificación en cuanto a tipo de tratamiento antibiótico empírico recibido, o en cuanto a la duración del mismo, o si supuso alguna actitud diagnóstica nueva tras el diagnóstico de bacteriemia, datos no analizados en este estudio. Si la respuesta a estos puntos es negativa, es decir el diagnóstico de bacteriemia no supuso ninguna modificación en el tratamiento recibido o en actitudes diagnósticas posteriores, la pregunta más adecuada sería plan- 
TABLA I

GÉRM ENES CLASIFICADOS POR M EDIO DE CRECIM IENTO AERO BIO-ANAERO BIO Y VALORACIÓN CLÍNICA

\begin{tabular}{|c|c|c|c|c|}
\hline \multirow[t]{2}{*}{ Germen } & \multicolumn{2}{|c|}{ Negativo y/o contaminante } & \multicolumn{2}{|c|}{ Verdadero positivo con o sin contaminante } \\
\hline & Cultivo aerobio & Cultivo anaerobio & Cultivo aerobio & Cultivo anaerobio \\
\hline No germen & $9(20 \%)$ & $45(95,7 \%)$ & & \\
\hline Staphylococcus sp coagulasa negativo & $25(55,6 \%)$ & $2(4,3 \%)$ & $1(4 \%)$ & $1(4,3 \%)$ \\
\hline Staphylococcus aureus & $1(2,2 \%)$ & & $1(4 \%)$ & $1(4,3 \%)$ \\
\hline Streptococcus acidominimus & & & $1(4 \%)$ & \\
\hline Streptococcus pyogenes & & & $1(4 \%)$ & $1(4,3 \%)$ \\
\hline Streptococcus anginosus & $1(2,2 \%)$ & & & \\
\hline Streptococcus pneumoniae & & & $4(16 \%)$ & $2(8,7 \%)$ \\
\hline Streptococcus pneumoniae+ Staphylococcus sp coagulasa negativ & & & $1(4 \%)$ & $1(4,3 \%)$ \\
\hline Escherichia coli & & & $12(48 \%)$ & $12(52,2 \%)$ \\
\hline Escherichia coli+Enterococcus faecalis & & & $1(4 \%)$ & $1(4,3 \%)$ \\
\hline Klebsiella sp+Staphylococcus sp coagulasa negativo & & & & $1(4,3 \%)$ \\
\hline Klebsiella sp+Bacillus sp & & & $1(4 \%)$ & \\
\hline Corynebacterium sp + Staphylococcus sp coagulasa negativo & $1(2,2 \%)$ & & & \\
\hline Corynebacterium sp & $1(2,2 \%)$ & & & \\
\hline Propionybacterium sp & $1(2,2 \%)$ & & & \\
\hline Micrococcus sp & $4(8,9 \%)$ & & $1(4 \%)$ & \\
\hline M icrococcus sp+ Staphylococcus sp coagulasa negativo & $1(2,2 \%)$ & & & \\
\hline Neisseria meningitidis & & & & $1(4,3 \%)$ \\
\hline Neiseria meningitidis+Streptococcus oralis & & & $1(4 \%)$ & \\
\hline Peptoestreptococcus & & & & $1(4,3 \%)$ \\
\hline Bacteroides sp & & & & $1(4,3 \%)$ \\
\hline Gemella sp & $1(2,2 \%)$ & & & \\
\hline TOTAL & $45(100 \%)$ & $47(100 \%)$ & $25(100 \%)$ & $23(100 \%)$ \\
\hline
\end{tabular}

Los porcentajes están dados por columnas.

tearse si estaban o no indicados la realización de los hemocultivos, pero una vez tomada la decisión de su extracción, dados los datos expuestos, deberían de realizarse hemocultivos tanto en frasco de aerobios como de anaerobios. Un segundo punto llamativo es el tipo de gérmenes que han presentado crecimiento en medio de cultivo para anaerobios.y que son mayoritariamente aerobios-anaerobios facultativos que representan la mayoría de estos crecimientos, e incluyen Enterobacteriaceae, estafilococos, y estreptococos. Es decir, tan sólo hay dos bacteriemias por anaerobios con crecimiento en frasco de anaerobios, frente a 21 bacteriemias por otros gérmenes que potencialmente son susceptibles de crecer en medio de crecimiento para aerobios y anaerobios. Ante este dato, la pregunta a realizarse sería la posible utilidad de la sustitución de los frascos de anaerobios por frascos de aerobios, dejando sólo el frasco de anaerobios en caso de sospecha de bacteriemia por anaerobios. A este respecto, el estudio realizado por Morris y cols. (6) sugiere un incremento del $6 \%$ en los aislamientos clínicamente significativos si un frasco de anaerobios se incluye con dos frascos de aerobios en casos clínicamente sugestivos de bacteriemias por anaerobios, apoyando la idea de incluir los frascos de anaerobios sólo en casos clínicamente sugestivos. Este dato vendría además apoyado por el hecho de que las bacteriemia por anaerobios representan una minoría dentro del conjunto global de las bacteriemias (3\%) (7-9). Sin embargo otros autores encuentran una pérdida de hasta el $15 \%$ de aislamientos significativos cuando no se incluyen frascos de anaerobios y sólo se realiza extracción en medio de cultivos para aerobios (10). La puntualización a este último dato sería si la pérdida de aislamientos significativos depende más de la pérdida de volumen de extracción al no hacer extracción en medios para anaerobios, que de la propia rentabilidad del medio de cultivo para anaerobios, es decir si se sustituyera la extracción de anaerobios por otra de aerobios, manteniendo el mismo volumen total de extracción, ¿se mantendría la rentabilidad de los hemocultivos, dada además la baja incidencia de bacteriemias por anaerobios?, como afirman algunos autores como James y cols. $(11,12)$. Así pues, en función de los datos obtenidos en nuestro estudio, la recomendación es la de mantener los frascos de hemocultivos para anaerobios, ya que la combinación medio de cultivo aerobio-anaerobio, aumenta de forma significativa la rentabilidad de los hemocultivos, y este hecho no es debido sólo a la detección de gérmenes anaerobios estrictos, sino que incluye además aerobios-anaerobios facultativos. Si estos resultados se deben al propio medio de cultivo, o al aumento de volumen de extracción, deberá de ser analizado en posteriores estudios, de los cuales en el momento actual existen pocos datos. 


\section{Bibliografía}

1. Lee CS, Hwang B, Chung RL, Tang RB. The assessment of anaerobic blood culture in children. J Microbiol Immunol Infect 2000; 33 (1): 49-52.

2. Ortiz E, Sande MA .Routine use of anaerobic blood cultures: are they still indicated? Am J Med 2000; 108 (6): 505-6.

3. Pottumarthy S, Morris AJ. Assessment of the yield of anaerobic blood cultures. Pathology 1997; 29 (4): 415-7.

4. Rosenblatt JE. Can we afford to do anaerobic cultures and identification? A positive point of view. Clin Infect Dis 1997; 25 (Supl. 2): S127-31.

5. Cockerill FR, Hughes JG, Vetter EA, Mueller RA, et al. Analysis of 281,797 consecutive blood cultures performed over an eight-year period: trends in microorganisms isolated and the value of anaerobic culture of blood. Clin Infect Dis 1997; 24 (3): 403-18.

6. Morris AJ, Wilson ML, Mirrett S, Reller LB. Rationale for selective use of anaerobic blood cultures. Journal of Clinical Microbiology 1993; 31: 2110-3.

7. Murray PR, Traynor P, Hopson D. Critical assessment of blood cultures techniques: Analysis of recovery of obligate and facultative anaerobes, strict aerobic bacteria and fungi in aerobic and anaerobic blood cultures bottles. Journal of Clinical Microbiology 1992; 30: 1462-8.

8. Sharp S. Routine anaerobic blood cultures: Still appropriate today? Clinical Microbiology Newsletter 1991; 13: 179-81.

9. Chandler MT, Morton ES, Byrd RP, Fields C, Roy MT, James H. Reevaluation of anaerobic blood cultures in a Veteran Population. South Med J 2000; 93 (10): 986-8.

10. Khanna P, Collignon P. Anaerobic bottles are still important in blood cultures sets. Eur J Clin Microbiol Infect Dis 2001; 20 (3): 217-9.

11. James PA, el Shafi KM. C. Clinical value of anaerobic blood cultures: a retrospective analysis of positive patient episodes. J Clin Pathol 2000; 53 (3): 231-3 .

12. Ziegler R, Johnscher I, Martus P, Lenhardt D, Just HM. Controlled clinical laboratory comparison of two supplemented aerobic and anaerobic media used in automated blood culture systems to detect bloodstream infections. J Clin Microbiol 1998; 36 (3): 657-61. 